\title{
Epigenetic basis of cancer drug resistance
}

\author{
Moammir H. Aziz', Aamir Ahmad² \\ 1James H. Quillen VA Medical Center, Johnson City, TN 37604, USA. \\ ${ }^{2}$ School of Medicine, University of Alabama at Birmingham, Birmingham, AL 35205, USA.
}

Correspondence to: Dr. Aamir Ahmad, School of Medicine, University of Alabama at Birmingham, 901 19th St S, 370 BMRII, Birmingham, AL 35205, USA. E-mail: aamirahmad@uabmc.edu How to cite this article: Aziz MH, Ahmad A. Epigenetic basis of cancer drug resistance. Cancer Drug Resist2020;3:113-6.
http://dx.doi.org/10.20517/cdr.2020.06

Received: 23 Jan 2020 Accepted: 31 Jan 2020 Published: 19 Mar 2020

Science Editor: Frits Peters Copy Editor: Jing-Wen Zhang Production Editor: Jing Yu

Cancer is a deadly disease and resistance to therapies is a major reason that renders it particularly lethal. A majority of research thus far has focused on genetic factors that form the basis of cancer drug resistance. However, it is increasingly being realized that epigenetic regulation plays a very important role in determining the resistance of individual tumors to certain therapies. Several novel drugs that target epigenetic events are under investigation, thus serving as a testimony to the enormous potential of exploiting epigenetics in tackling the problem of cancer drug resistance. Moving forward, a focus on epigenetics will be critical for a better understanding of the phenomenon of cancer drug resistance and for the design of novel therapies.

Some cancer patients are inherently resistant to specific therapy because of their genetic makeup (de novo cancer drug resistance) while other cancer patients initially respond to therapy, but eventually develop resistance with continued administration (acquired cancer drug resistance) ${ }^{[1]}$. A good understanding of cancer drug resistance is critical to the efficient management of cancer patients in the clinics. Epigenetic events have a profound effect on the onset as well as progression of human cancers ${ }^{[2,3]}$. Methylation and acetylation are two well-studied epigenetic events that are known to profoundly affect the expression of genes, resulting in activation of oncogenes and/or suppression of tumor suppressor genes, leading to development of cancer drug resistance. DNA methylation, histone modifications (methylation, acetylation, phosphorylation, ubiquitylation, sumoylation, etc.), and regulation through microRNAs (miRNAs) are some of the active areas of cancer research, encompassing the epigenetic regulation (see the Special Issue on: Non-coding RNAs in Response and Resistance to Therapy in Cancer). 
The tyrosine kinase inhibitors (TKIs) have an important role in cancer therapy. They are effective against many cancers, both solid and liquid. They have particularly been used to inhibit the oncogenic activity associated with epidermal growth factor receptor (EGFR), a receptor tyrosine kinase. However, acquired resistance against EGFR-TKIs is well-studied ${ }^{[4]}$ with reports on its epigenetic regulation ${ }^{[5,6]}$. White et al. ${ }^{[7]}$ reviewed the subject by evaluating a role of histone lysine demethylases in resistance against TKIs. Upregulation of these demethylases leads to resistance against TKIs. For example, amplification of KDM5A gene in breast cancer correlates with erlotinib resistance, as well as with gefitinib resistance in lung cancer. Erlotinib and gefitinib are first generation EGFR-TKIs. Similarly, KDM1A, also known as lysinespecific histone demethylase $1 \mathrm{~A}$, mediates resistance against sorafenib in liver cancer cells. Sorafenib is a kinase inhibitor effective against rapidly accelerated fibrosarcoma, vascular endothelial growth factor receptor, and platelet-derived growth factor receptor kinases. Based on this evidence, inhibition of lysine demethylases can be an effective strategy to overcome resistance against TKIs.

Ovarian cancer is the most lethal gynecological cancer, with a reported epigenetic basis of drug resistance ${ }^{[8]}$. Platinum-based anticancer drugs are commonly used to treat ovarian cancer but the tumors often progress within the first two years of treatment. Schwarzenbach and Gahan ${ }^{[9]}$ discussed the epigenetic signatures, such as DNA methylation, histone modifications, and miRNA deregulation, in cis- or carboplatin resistant ovarian cancers. Such knowledge can help identify patients who will respond favorably to platinumbased therapies $v$ s. those who will not. This is a step towards personalized medicine in cancer, a concept that is increasingly attracting the attention of cancer researchers ${ }^{[10-12]}$. Breast cancer is another cancer that is frequently diagnosed in females and which remains a leading cause of cancer-related deaths in females worldwide. Among the many mechanisms, epithelial-to-mesenchymal transition (EMT) and the acquisition of cancer stem-like phenotype provide plasticity to breast cancer cells, rendering these cells highly invasive, metastatic, and resistant to therapies. The article by Ponnusamy et al. ${ }^{[13]}$ provides a better understanding of epigenetic modifications underpinning the cellular reprogramming, particularly the EMT and stem cell phenotype. Such knowledge is essential to design future therapies to overcome cancer drug resistance. On a similar topic, the research article by Seno et al ${ }^{[14]}$ describes the cytotoxic action of daunorubicin, a topoisomerase II inhibitor, against mouse induced pluripotent stem cells-derived cancer stem cells.

The relevance of epigenetic events is not limited to solid tumors. In acute lymphoblastic leukemia, a common childhood malignancy, there is an increasing realization that genetic changes are not enough to describe the observed chemoresistance and the resulting relapse. As a result, researchers have started looking at the epigenetic changes as a possible answer to this problem ${ }^{[15]}$ and this is the topic of discussion in the article by Meyer et al. ${ }^{[16]}$. Further, a cell organelle that is relatively less studied in the context of epigenetic regulation of drug resistance is the centrosome. The centrosome plays a very important role during cell division, ensuring proper distribution of cellular components among daughter cells. Any aberrant functioning of centrosomes, through epigenetic events, can compromise the integrity of cells, resulting in genetic instability. The article by Jia et al. ${ }^{[17]}$ touches upon this important topic of understanding epigenetic changes in the centrosome as relevant to cancer drug resistance.

The focus on immune cells-cancer cells interactions and the reprograming of cancer cells enabling evasion of host immune system over the past several years has brought cancer immunology to the forefront of cancer research $^{[18]}$. In particular, immune checkpoints block the ability of the immune system to act on cancer cells. Thus, immune checkpoint inhibitors have been evaluated to boost up the immune system and potentiate T-cells to recognize and attack tumor cells. As seen in almost all anti-cancer therapies, resistance against immune checkpoint inhibitors has also been reported. Peixoto et al. ${ }^{[19]}$ described the epigenetic modifications that underline the resistance against immunotherapy. 
Although methylation and acetylation are classical epigenetic events, regulation through miRNAs is also considered an example of epigenetic modification. Further, because of the importance of miRNAs in the regulation of several physiological processes, their expression is itself tightly controlled, often through epigenetic regulation ${ }^{[20-22]}$, thus making miRNAs an important part of cellular epigenetic machinery. The article by Biersack ${ }^{[23]}$ touches upon the role of miRNAs in resistance to alkylating anticancer agents, with a focus on both natural and synthetic alkylating agents and their interactions with miRNAs.

In summary, this Special Issue on the epigenetic basis of cancer drug resistance touches upon some very diverse and hot topics that detail the current progress in understanding the importance of epigenetic events in determining the resistance against various therapies and in a number of different cancers. Without such understanding, the design of novel therapies to counter drug resistance will be impossible.

\section{DECLARATIONS}

\section{Authors' contributions}

Drafted and proof-read the article: Aziz MH, Ahmad A

\section{Availability of data and materials}

Not applicable.

\section{Financial support and sponsorship}

None.

\section{Conflicts of interest}

All authors declared that there are no conflicts of interest.

\section{Ethical approval and consent to participate}

Not applicable.

\section{Consent for publication}

Not applicable.

\section{Copyright}

(c) The Author(s) 2020.

\section{REFERENCES}

1. Peters GJ. Cancer drug resistance: a new perspective. Cancer Drug Resist 2018;1:1-5.

2. Ahmad A. Editorial: cancer epigenetics. Curr Cancer Drug Targets 2018;18:3-4.

3. Ahmad A. Cancer epigenetics: clinical perspectives. Curr Cancer Drug Targets 2019;19:513-4.

4. Ahmad A, Maitah MY, Ginnebaugh KR, Li Y, Bao B, et al. Inhibition of Hedgehog signaling sensitizes NSCLC cells to standard therapies through modulation of EMT-regulating miRNAs. J Hematol Oncol 2013;6:77.

5. Lu Y, Liu Y, Oeck S, Glazer PM. Hypoxia promotes resistance to EGFR inhibition in NSCLC Cells via the histone demethylases, LSD1 and PLU-1. Mol Cancer Res 2018;16:1458-69.

6. Yue J, Lv D, Wang C, Li L, Zhao Q, et al. Epigenetic silencing of miR-483-3p promotes acquired gefitinib resistance and EMT in EGFR-mutant NSCLC by targeting integrin beta3. Oncogene 2018;37:4300-12.

7. White JC, Pucci P, Crea F. The role of histone lysine demethylases in cancer cells' resistance to tyrosine kinase inhibitors. Cancer Drug Resist 2019;2:326-34.

8. Moufarrij S, Dandapani M, Arthofer E, Gomez S, Srivastava A, et al. Epigenetic therapy for ovarian cancer: promise and progress. Clin Epigenetics 2019;11:7.

9. Schwarzenbach H, Gahan PB. Resistance to cis- and carboplatin initiated by epigenetic changes in ovarian cancer patients. Cancer Drug Resist 2019;2:271-96.

10. Ahmad A. Epigenetics in personalized management of lung cancer. Adv Exp Med Biol 2016;890:111-22. 
11. Malone ER, Oliva M, Sabatini PJB, Stockley TL, Siu LL. Molecular profiling for precision cancer therapies. Genome Med 2020;12:8.

12. Goutsouliak K, Veeraraghavan J, Sethunath V, Angelis CD, Osborne CK, et al. Towards personalized treatment for early stage HER2positive breast cancer. Nat Rev Clin Oncol 2019; Epub ahead of print. doi:10.1038/s41571-019-0299-9.

13. Ponnusamy L, Mahalingaiah PKS, Chang YW, Singh KP. Role of cellular reprogramming and epigenetic dysregulation in acquired chemoresistance in breast cancer. Cancer Drug Resist 2019;2:297-312.

14. Seno A, Mizutani A, Aizawa K, Onoue R, Masuda J, et al. Daunorubicin can eliminate iPS-derived cancer stem cells via ICAD/CADindependent DNA fragmentation. Cancer Drug Resist 2019;2:335-50.

15. Janczar S, Janczar K, Pastorczak A, Harb H, Paige AJW, et al. The role of histone protein modifications and mutations in histone modifiers in pediatric B-cell progenitor acute lymphoblastic leukemia. Cancers (Basel) 2017;9.

16. Meyer LK, Hermiston ML. The epigenome in pediatric acute lymphoblastic leukemia: drug resistance and therapeutic opportunities. Cancer Drug Resist 2019;2:313-25.

17. Jia ZH, Wang XG, Zhang H. Overcome cancer drug resistance by targeting epigenetic modifications of centrosome. Cancer Drug Resist 2019;2:210-24.

18. Galon J, Bruni D. Tumor immunology and tumor evolution: intertwined histories. Immunity 2020;52:55-81.

19. Peixoto P, Renaude E, Boyer-Guittaut M, Hervouet E. Epigenetics, a key player of immunotherapy resistance. Cancer Drug Resist 2018;1:219-29.

20. Morales S, Monzo M, Navarro A. Epigenetic regulation mechanisms of microRNA expression. Biomol Concepts 2017;8:203-12.

21. Ahmad A, Li Y, Bao B, Kong D, Sarkar FH. Epigenetic regulation of miRNA-cancer stem cells nexus by nutraceuticals. Mol Nutr Food Res 2014;58:79-86.

22. Ediriweera MK, Cho SK. Targeting miRNAs by histone deacetylase inhibitors (HDACi): Rationalizing epigenetics-based therapies for breast cancer. Pharmacol Ther 2019;206:107437.

23. Biersack B. Alkylating anticancer agents and their relations to microRNAs. Cancer Drug Resist 2019;2:1-17. 